\title{
Correction to: The association between socioeconomic disadvantage and parent-rated health in children and adolescents with chronic kidney disease - the Kids with CKD (KCAD) study
}

\author{
Madeleine Didsbury ${ }^{1,2} \cdot$ Anita van Zwieten ${ }^{1,2} \cdot$ Kerry Chen $^{2} \cdot$ Laura J. James $^{1,2} \cdot$ Anna Francis $^{1,2,3} \cdot$ Siah Kim $^{1,2}$. \\ Steven McTaggart ${ }^{3} \cdot$ Amanda Walker $^{4} \cdot$ Fiona Mackie $^{5} \cdot$ Tonya Kara $^{6} \cdot$ Chanel Prestidge $^{6}$. Armando Teixeira-Pinto ${ }^{1,2}$. \\ Belinda Barton ${ }^{7}$. Jennifer Lorenzo ${ }^{8}$. Suncica Lah ${ }^{9,10} \cdot$ Kirsten Howard $^{2}$ - Natasha Nassar ${ }^{11}$ - Eric $A u^{1,2}$. \\ Allison Tong ${ }^{1,2} \cdot$ Jonathan C. Craig ${ }^{1,2} \cdot$ Germaine Wong ${ }^{1,2,12}$
}

Published online: 10 January 2022

(c) The Author(s), under exclusive licence to International Pediatric Nephrology Association 2021

Correction to: Pediatr Nephrol (2019) 34:1237 - 1245 https://doi.org/10.1007/s00467-019-04209-7

In this paper: The association between socioeconomic disadvantage and parent-rated health in children and adolescents with chronic kidney disease - the Kids with CKD (KCAD) study, the authors noticed a number of numerical errors while they rechecked their analyses. These do not affect their overall conclusions.

These changes impact results in Fig. 3 and Tables 1, 2, 3,4 .

In the estimates for the 'Association between socioeconomic disadvantage and parent-rated health' in the abstract, it should read "In the entire cohort, the adjusted odds ratios (ORs) and 95\% confidence intervals (CIs) for poor parent-rated health were 1.73 (1.03-2.92) for lower

The original article can be found online at https://doi.org/10.1007/ s00467-019-04209-7.

Germaine Wong

germaine.wong@health.nsw.gov.au

1 Centre for Kidney Research, The Children's Hospital at Westmead, Sydney, Australia

2 Sydney School of Public Health, The University of Sydney, Sydney, NSW 2006, Australia

3 Child \& Adolescent Renal Service, Lady Cilento Children's Hospital, Brisbane, Australia

4 Department of Renal Medicine, The Royal Children's Hospital, Melbourne, Australia

5 Department of Nephrology, Sydney Children's Hospital at Randwick, Sydney, Australia

6 Department of Nephrology, Starship Children's Hospital, Auckland, New Zealand household income, $1.62(0.97-2.71)$ for families that did not own their own home, 2.58 (1.52-4.38) for caregivers who rated their financial status as poor, 0.75 (0.45-1.23) for lower educational attainment and 1.49 (0.90-2.47) for children whose primary caregivers were unemployed. With reference to the highest global SES index quartile, adjusted ORs for poor parent-rated health in descending order were 1.72 (0.77-3.82), 2.71 (1.25-5.88) and 2.43 (1.10-5.39), respectively. The association between low SES and poor parent-rated health was modified by CKD stage, where lower global SES index was independently associated with poor parent-rated health in children with CKD stages I-V, but not children on dialysis or with kidney transplants $(p=0.01)$."

This error was repeated in results paragraphs of the main text that refer to Fig. 3 and Tables 2-4. This also impacted the first line of the first paragraph of the discussion which

7 Children's Hospital Education Research Institute, The Children's Hospital at Westmead, Sydney, Australia

8 Institute for Neuroscience and Muscle Research, The Children's Hospital at Westmead, Sydney, Australia

9 School of Psychology, The University of Sydney, Sydney, Australia

10 The ARC Centre of Excellence in Cognition and Its Disorders, Macquarie University, Sydney, Australia

11 Sydney School of Public Health, Menzies Centre for Health Policy, The University of Sydney, Sydney, Australia

12 Centre for Transplant and Renal Research, Westmead Hospital, Sydney, Australia 


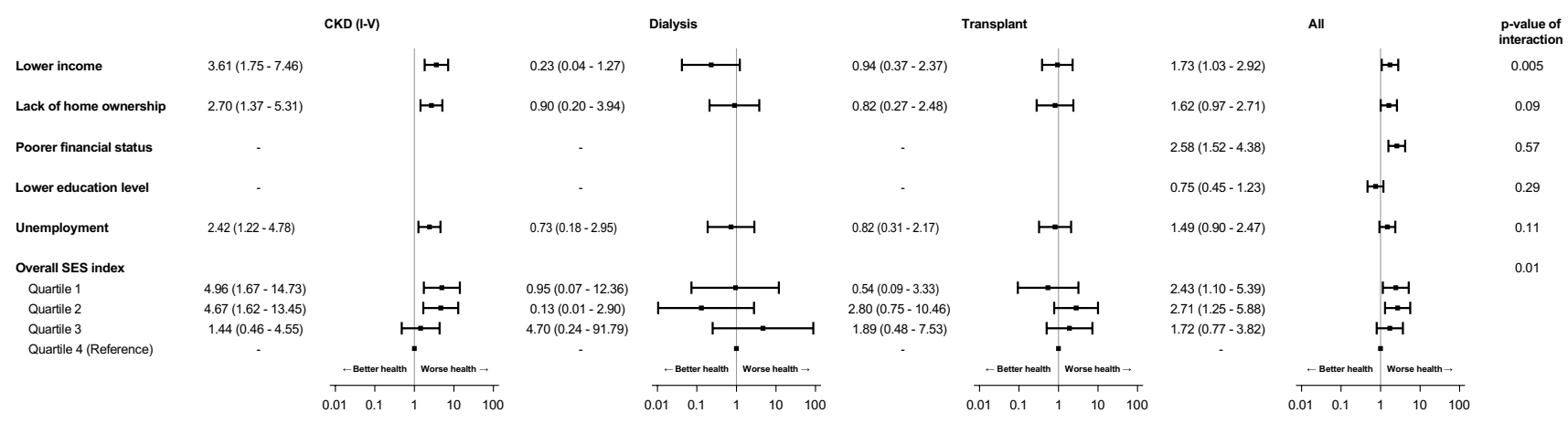

Fig. 3 Association between socioeconomic disadvantage and parentrated health of the child with CKD. Notes: Each odds ratio is from a separate regression, adjusted for age, ethnicity and gender (plus CKD stage for those in the "All" column). Reference categories for individual socioeconomic exposures (in order from top to bottom) are: higher income (> \$1250AUD/week), home ownership (owned outright/mortgage), better financial status (comfortable to prosperous), higher education level (other certificate or diploma/bachelor's degree

should read 'Children from lower SES backgrounds in terms of financial status were approximately 2.5 times as likely to experience fair or poor health compared to those of higher SES' and the third line of the first paragraph of the or higher/other education), any employment. Global SES index is a continuous variable derived from a principal component analysis on all 5 individual socioeconomic exposures, as described in the methods. It was categorized into quartiles for the regression, with Quartile 4 (highest socioeconomic status) treated as the reference category. The p-values are for the interaction between each socioeconomic exposure and CKD stage (CKD I-V vs. dialysis vs. transplant).

discussion. 'Children with CKD stages $1-V$, but not children on renal replacement therapy (RRT) (dialysis and with kidney transplants), whose caregivers were either unemployed, had below average weekly income or lack of home ownership

Table 1 Baseline characteristics of children with CKD and their caregivers $(\mathrm{N}=377)$

\begin{tabular}{|c|c|c|c|c|c|c|c|c|c|}
\hline \multirow[b]{2}{*}{ Characteristics of childre } & \multicolumn{2}{|c|}{$\begin{array}{l}\text { Stage I-V } \\
\mathrm{N}=199 \\
\mathrm{~N}(\%)\end{array}$} & \multicolumn{3}{|c|}{$\begin{array}{l}\text { Dialysis } \\
N=43 \\
\mathrm{~N}(\%)\end{array}$} & \multicolumn{2}{|c|}{$\begin{array}{l}\text { Transplant } \\
\mathrm{N}=135 \\
\mathrm{~N}(\%)\end{array}$} & \multicolumn{2}{|c|}{$\begin{array}{l}\text { Total } \\
\mathrm{N}=377 \\
\mathrm{~N}(\%)\end{array}$} \\
\hline & & & & & & & & & \\
\hline \multicolumn{10}{|l|}{ Gender } \\
\hline Male & 133 & (67) & 21 & (49) & & 79 & (59) & 233 & (62) \\
\hline \multicolumn{10}{|l|}{ Age (years) } \\
\hline $6-9$ & 68 & (34) & 11 & (26) & & 41 & (30) & 120 & (32) \\
\hline $10-14$ & 83 & (42) & 16 & (37) & & 43 & (32) & 142 & (38) \\
\hline $15+$ & 48 & (24) & 16 & (37) & & 51 & (38) & 115 & (31) \\
\hline \multicolumn{10}{|l|}{ Cause of CKD } \\
\hline CAKUT & 62 & (31) & 18 & (42) & & 47 & (35) & 127 & (34) \\
\hline Glomerulonephritis & 34 & (17) & 6 & (14) & & 18 & (13) & 58 & (15) \\
\hline Nephrotic & 59 & (30) & 6 & (14) & & 27 & (20) & 92 & (24) \\
\hline Cystic & 14 & (7) & 4 & (9) & & 13 & (10) & 31 & (8) \\
\hline Other & 30 & (15) & 9 & (21) & & 30 & (22) & 69 & (18) \\
\hline \multicolumn{10}{|l|}{ Ethnicity } \\
\hline Australian Indigenous & 8 & (4) & 2 & (5) & & 7 & (5) & 17 & (5) \\
\hline Māori & 3 & (2) & 2 & (5) & & 2 & (1) & 7 & (2) \\
\hline Pacific Islander & 1 & (1) & 2 & (5) & & 1 & (1) & 4 & (1) \\
\hline Caucasian & 108 & (54) & 24 & (56) & & 88 & (65) & 220 & (58) \\
\hline Middle Eastern & 22 & (11) & 7 & (16) & & 13 & (10) & 42 & (11) \\
\hline Asian & 40 & (20) & 2 & (5) & & 16 & (12) & 58 & (15) \\
\hline Other & 14 & (7) & 3 & (7) & & 5 & (4) & 22 & (6) \\
\hline \multicolumn{10}{|l|}{ Private Health Insurance } \\
\hline Yes & 78 & (39) & 7 & (16) & 50 & & (37) & 135 & (36) \\
\hline \multicolumn{10}{|l|}{ State/country } \\
\hline New South Wales & 142 & (71) & 15 & (35) & 73 & & (54) & 230 & (61) \\
\hline Queensland & 5 & (3) & 9 & (21) & 15 & & (11) & 29 & (8) \\
\hline
\end{tabular}


Table 1 (continued)

\begin{tabular}{|c|c|c|c|c|c|c|c|c|c|}
\hline \multirow[b]{2}{*}{ Victoria } & \multicolumn{2}{|c|}{$\begin{array}{l}\text { Stage I-V } \\
\mathrm{N}=199 \\
\mathrm{~N}(\%)\end{array}$} & \multicolumn{2}{|c|}{$\begin{array}{l}\text { Dialysis } \\
\mathrm{N}=43 \\
\mathrm{~N}(\%)\end{array}$} & & \multicolumn{2}{|c|}{$\begin{array}{l}\text { Transplant } \\
\mathrm{N}=135 \\
\mathrm{~N}(\%)\end{array}$} & \multicolumn{2}{|c|}{$\begin{array}{l}\text { Total } \\
\mathrm{N}=377 \\
\mathrm{~N}(\%)\end{array}$} \\
\hline & 22 & (11) & 15 & (35) & 30 & & (22) & 67 & $(18)$ \\
\hline New Zealand & 30 & (15) & 4 & (9) & 17 & & (13) & 51 & (14) \\
\hline \multicolumn{10}{|l|}{ Characteristics of caregivers } \\
\hline \multicolumn{10}{|l|}{ Age } \\
\hline$<40$ years & 56 & (28) & 15 & (35) & & 41 & (30) & 112 & (30) \\
\hline $40-70$ years & 138 & $(69)$ & 27 & (63) & & 91 & (67) & 256 & $(68)$ \\
\hline \multicolumn{10}{|l|}{ Gender } \\
\hline Male & 30 & $(15)$ & 9 & (21) & & 17 & (13) & 56 & (15) \\
\hline \multicolumn{10}{|l|}{ Education } \\
\hline Primary school/secondary school/trade certificate & 76 & $(38)$ & 24 & (56) & & 60 & (44) & 160 & $(42)$ \\
\hline $\begin{array}{l}\text { Other certificate or diploma/other education/ Bachelor's degree } \\
\text { or higher }\end{array}$ & 123 & (62) & 18 & $(42)$ & & 74 & $(55)$ & 215 & $(57)$ \\
\hline \multicolumn{10}{|l|}{ Household income } \\
\hline$<\$ 1250 \mathrm{AUD} / \mathrm{wk}$ & 88 & $(44)$ & 26 & (60) & & 69 & $(51)$ & 183 & (49) \\
\hline$>\$ 1250 \mathrm{AUD} / \mathrm{wk}$ & 103 & (52) & 12 & (28) & & 58 & (43) & 173 & (46) \\
\hline *Prefer not to say & 8 & (4) & 5 & (12) & & 8 & (6) & 21 & (6) \\
\hline \multicolumn{10}{|l|}{ Employment status } \\
\hline Unemployed & 79 & (40) & 25 & (58) & & 52 & (39) & 156 & (41) \\
\hline Any employment & 120 & $(60)$ & 17 & (40) & & 82 & (61) & 219 & $(58)$ \\
\hline \multicolumn{10}{|l|}{ Marital status } \\
\hline Single & 34 & (17) & 12 & (28) & & 31 & (23) & 77 & (20) \\
\hline Married/defacto & 165 & $(83)$ & 29 & $(67)$ & & 102 & (76) & 296 & (79) \\
\hline \multicolumn{10}{|l|}{ Perceived financial status } \\
\hline Very poor to getting along & 100 & $(50)$ & 31 & (72) & & 72 & (53) & 203 & (54) \\
\hline Comfortable to prosperous & 99 & $(50)$ & 10 & (23) & & 62 & (46) & 171 & (45) \\
\hline \multicolumn{10}{|l|}{ Home ownership } \\
\hline Rented/other & 69 & (35) & 16 & (37) & & 32 & (24) & 117 & (31) \\
\hline Owned outright/mortgage & 129 & $(65)$ & 25 & $(58)$ & & 101 & (75) & 255 & (68) \\
\hline
\end{tabular}

Notes: Caregiver characteristics displayed are of the caregiver who filled out the questionnaire. Percentages displayed of total including missing data: caregiver age, $n=9(2 \%)$, caregiver gender $n=2(0.5 \%)$, private health insurance $n=1(0.3 \%)$, ethnicity $n=7(2 \%)$, education $n=2(0.5 \%)$, employment $n=2(0.5 \%)$, marital status $n=4(1 \%)$, financial status $n=3(1 \%)$, home ownership $n=5(1 \%)$. *indicative of question refusal.

were approximately 2.5 to 3.5 times as likely to experience fair to poor health compared to children with caregivers who were more affluent across all these socioeconomic measures.' Similarly, in the final paragraph of the discussion the second sentence should read "This is influenced by CKD stage whereby among children with stage I-V (but not those on RRT), low global SES was associated with approximately 1.5 to 5 times the odds of poor parent-rated child health across decreasing SES quartiles."
In the second and third paragraph of results, this should read 'The median age of participating primary caregivers was 43.3 years [IQR 38.9-48.2], 77 (20\%) were single parents, 156 (41\%) were not in paid employment, 215 (57\%) had certificate/diploma, other education, bachelor's degree or higher tertiary education, and 255 (68\%) owned property either outright or with a mortgage. A higher proportion of caregivers of children on dialysis were unemployed $(p=0.01)$, in the lower income category $(p=0.03)$ and
Table 2 Correlation matrix of the key socioeconomic determinants of the cohort

\begin{tabular}{llllll}
\hline & Income & $\begin{array}{l}\text { Perceived finan- } \\
\text { cial status }\end{array}$ & Employment & $\begin{array}{l}\text { Home own- } \\
\text { ership }\end{array}$ & Education \\
\hline Income & 1 & 0.48 & 0.40 & 0.37 & 0.34 \\
Perceived financial status & 0.48 & 1 & 0.26 & 0.29 & 0.19 \\
Employment & 0.40 & 0.26 & 1 & 0.14 & 0.23 \\
Home ownership & 0.37 & 0.29 & 0.14 & 1 & 0.19 \\
Education & 0.34 & 0.19 & 0.23 & 0.19 & 1 \\
\hline
\end{tabular}


Table 3 Results from the principal component analyses

\begin{tabular}{lllll}
\hline Component & Eigenvalue & Differences & $\begin{array}{l}\text { Variance } \\
\text { propor- } \\
\text { tion }\end{array}$ & $\begin{array}{l}\text { Cumulative } \\
\text { variance pro- } \\
\text { portion }\end{array}$ \\
\hline Component 1 & 2.21 & 1.33 & 0.44 & 0.44 \\
Component 2 & 0.88 & 0.09 & 0.18 & 0.62 \\
Component 3 & 0.79 & 0.13 & 0.16 & 0.78 \\
Component 4 & 0.66 & 0.20 & 0.13 & 0.91 \\
Component 5 & 0.46 & & 0.09 & 1.0 \\
\hline
\end{tabular}

Table 4 Contribution of variables to component 1 from principal component analyses

\begin{tabular}{ll}
\hline Variables & $\begin{array}{l}\text { Factor loadings } \\
\text { for component 1 }\end{array}$ \\
\hline Income & 0.82 \\
Perceived financial status & 0.70 \\
Employment & 0.61 \\
Home ownership & 0.59 \\
Education & 0.57 \\
\hline
\end{tabular}

reported lower perceived financial status than caregivers whose children were not on dialysis $(p=0.005)$, while caregivers of children on dialysis were marginally more likely to have lower education levels $(p=0.05)$, and there were no differences in home ownership $(p=0.27)$.'

'After adjusting for age and gender, children on dialysis had 2.6 times the odds of poorer parent-rated health than those with CKD stages I-V or after transplant [adjusted OR (95\% CI): 2.57 (1.32-5.01), $\mathrm{p}=0.006]$. Adjusting for CKD stage, children were more likely to experience poorer parent-rated health if they had any comorbidities [adjusted OR (95\% CI): 3.15 (1.73-5.74), $\mathrm{p}<0.001$ ]. In particular, the likelihood of experiencing poor parent-rated health was higher among those with hypertension [adjusted OR (95\% CI): 1.77 (1.06-2.97)], growth deficiency [1.81 (1.04-3.14)] and chronic infections [3.82 (1.57-9.29)]. Parents with poor selfrated health were also more likely to rate their child's health as poor or fair, after adjustment for CKD stage [adjusted OR (95\% CI): 3.16 (1.69-5.90), $\mathrm{p}<0.001]$.'

Publisher's note Springer Nature remains neutral with regard to jurisdictional claims in published maps and institutional affiliations. 